Published in final edited form as:

Biol Psychiatry. 2010 February 1; 67(3): 279-282. doi:10.1016/j.biopsych.2009.08.036.

\title{
Genome-wide association study of movement-related adverse antipsychotic effects
}

\author{
Karolina Åberga ${ }^{a},{ }^{\star}$ Daniel E. Adkins ${ }^{a}$, József Bukszára, Bradley T. Webb ${ }^{a}$, Stanley N. \\ Caroff $^{b}$, Del D. Miller ${ }^{c}$, Jonathan Sebat ${ }^{d}$, Scott Stroup ${ }^{e}$, Ayman H. Fanous ${ }^{f, g, h}$, Vladimir I. \\ Vladimirov $^{\dagger}$, Joseph L. McClay ${ }^{a}$, Jeffrey A. Lieberman', Patrick F. Sullivan ${ }^{j}, k$, and Edwin \\ J.C.G. van den Oord ${ }^{a}$ \\ ${ }^{a}$ Center for Biomarker Research and Personalized Medicine, School of Pharmacy, Medical \\ College of Virginia of Virginia Commonwealth University, Richmond VA \\ bUniversity of Pennsylvania School of Medicine and the Department of Veterans Affairs Medical \\ Centre, Philadelphia, PA \\ 'University of lowa Carver College of Medicine, lowa City, IA \\ dCold Spring Harbor Laboratory, Cold Spring Harbor, NY \\ eDepartment of Psychiatry, University of North Carolina at Chapel Hill, NC \\ fVirginia Institute for Psychiatric and Behavioral Genetics, Department of Psychiatry, Medical \\ College of Virginia of Virginia Commonwealth University, Richmond, VA \\ gWashington VA Medical Center, Washington, DC \\ hDepartment of Psychiatry, Georgetown University Medical Center, Washington, DC \\ 'Department of Psychiatry, Columbia University, New York, NY \\ iDepartments of Genetics, Psychiatry, \& Epidemiology, University of North Carolina at Chapel Hill, \\ NC \\ kDepartment of Medical Epidemiology \& Biostatistics, Karolinska Institutet, Stockholm, Sweden
}

\begin{abstract}
Background-Understanding individual differences in the development of extra-pyramidal side effects (EPS) as a response to antipsychotic therapy is essential to individualize treatment.
\end{abstract}

Methods-We performed genome-wide association studies to search for genetic susceptibility to EPS. Our sample consists of 738 schizophrenia patients, genotyped for 492K SNPs. We studied three quantitative measures of antipsychotic adverse drug reactions, the Simpson-Angus scale (SAS) for parkinsonism, the Barnes akathisia rating scale, and the abnormal involuntary movement scale (AIMS) as well as a clinical diagnosis of probable tardive dyskinesia.

(C) 2009 Society of Biological Psychiatry. Published by Elsevier Inc. All rights reserved.

"Correspondence to: Karolina Åberg, Center for Biomarker Research and Personalized Medicine, School of Pharmacy, Medical College of Virginia of Virginia Commonwealth University, 1112 East Clay Street, P.O. Box 980533, Richmond, VA 23298. Tel: +1 804-628 3023, fax: +1 804-628 3991, kaaberg@ vcu.edu.

Publisher's Disclaimer: This is a PDF file of an unedited manuscript that has been accepted for publication. As a service to our customers we are providing this early version of the manuscript. The manuscript will undergo copyediting, typesetting, and review of the resulting proof before it is published in its final citable form. Please note that during the production process errors may be discovered which could affect the content, and all legal disclaimers that apply to the journal pertain. 
Results-Two SNPs for SAS, rs17022444 and rs2126709 with $p=1.2 \times 10^{-10}$ and $p=3.8 \times 10^{-7}$, respectively, and one for AIMS, rs7669317 with $p=7.7 \times 10^{-8}$, reached genome-wide significance $(q$-value $<0.1)$. Rs 17022444 and rs7669317 were located in intergenic regions and rs2126709 was located in $Z N F 202$ on 11q24. Fourteen additional signals were potentially interesting ( $q$-value $<0.5$ ). The $Z N F 202$ is a transcriptional repressor controlling, among other genes, $P L P 1$ which is the major protein in myelin. Mutations in $P L P 1$ cause Pelizaeus-Merzbacher disease, which has parkinsonism as an occurring symptom. Altered mRNA expression of $P L P 1$ is associated with schizophrenia.

Conclusions-Although our findings require replication and validation, this study demonstrates the potential of GWAS to discover genes and pathways that mediate adverse effects of antipsychotics.

\section{Keywords}

genome-wide association; antipsychotic; pharmacogenetics; personalized medicine; single nucleotide polymorphism; copy number variation; schizophrenia

\section{Introduction}

Antipsychotics are the cornerstone of acute and long-term treatment for schizophrenia. The first generation "typical" antipsychotics (e.g., haloperidol) were introduced in the 1950s. Despite treatment with these antipsychotics, many schizophrenia patients do not improve or relapse frequently. Furthermore, these drugs may produce adverse reactions such as extrapyramidal side effects (EPS, i.e., involuntary movements). Clozapine was (re-)introduced in 1989 marking the advent of the second generation "atypical" antipsychotics. Although clozapine has enhanced therapeutic effects in patients who respond poorly to treatment with typical antipsychotics, it is associated with a serious risk of agranulocytosis. Other second generation antipsychotics such as olanzapine have therefore become the first line treatment for schizophrenia. These second generation drugs are, however, no panaceas. They too lack efficacy in a substantial group of patients and are associated with, for example, metabolic side effects that increase risk of cardiovascular disease and diabetes. Due to effectiveness in individual patients, low risk of metabolic side effects, and much lower costs, first generation antipsychotics are therefore still being prescribed as well.

EPS are a major consideration for prescribing, in particular first generation antipsychotics that are assumed to have a somewhat higher risk profile. EPS are a complex and potentially heterogeneous group of involuntary movements including parkinsonism, akathisia, and tardive dyskinesia (TD). TD is particularly worrisome because of its high annual incidence (5.5\% and 3.95\% for first and second generation antipsychotics, respectively) and potential irreversibility (1). The complexity of EPS is illustrated by data showing that these side effects may occur at different times during antipsychotic treatment (acute vs. late EPS), may sometimes occur "spontaneously' after antipsychotic treatment ended, or may be suppressed by continued antipsychotic treatment. Clearly, a better understanding of individual differences in the susceptibility of developing EPS would be essential to better tailor antipsychotic treatment to individual patients.

Genetic factors have been proposed to explain part of the individual differences (2). Some candidate gene studies have explored the possible role of dopamine and serotonin receptors (3), and a recent meta-analysis supported an association with $D R D 2(4,5)$. However, because the selection of candidate genes is hampered by the limited knowledge of the causal mechanisms, exploratory methods that systematically screen markers across the whole genome for association with EPS are critical to discover novel variants. In this article, we 
therefore performed such a genome-wide association studies (GWAS) to search for genetic susceptibility to EPS as a result of cumulative lifetime antipsychotic exposure.

\section{Methods}

Methodological details can be found in the Supplemental Materials. In short, all subjects $(\mathrm{N}=738)$ came from the Clinical Antipsychotic Trial of Intervention Effectiveness (CATIE) study (6) and were diagnosed with schizophrenia according to DSM-IV. CATIE is a multiphase randomized trial of antipsychotic medications that recruited patients from 57 clinical settings around the United States. The mean age is 40.9 years. Approximately 57\% of the subjects describe themselves as white/European American (EA), 29\% as black/ African American (AA), and 14\% as belonging to multiple or "other" racial categories.

EPS was measured using the Simpson-Angus Scale (SAS) (7) for parkinsonism, the Barnes Akathisia Rating Scale (BARS) (8) and the Abnormal Involuntary Movement Scale (AIMS) (9). Patients with TD at initial assessment were excluded from assignment to perphenazine. Newly detected probable TD resulted in discontinuation of the current study treatment unless the research clinician suspected withdrawal TD, which according to the SchoolerKane criteria occurs within two weeks of discontinuing an antipsychotic medication.

On average CATIE subjects began antipsychotic treatment over 14 years ago. Our aim was to better understand genetic susceptibility to EPS as a result of this cumulative lifetime antipsychotic exposure. For this purpose, we first created a set of "proxy" indicators of lifetime exposure. Next, we used statistical modeling to measure the severity of EPS as a result of this exposure by essentially a) estimating the mean symptom level across the entire study using all rather than a single observation, while b) adjusting for subject-specific intrial changes. Finally, by including the proxies in the GWAS we searched for genetic variants predicting individual differences in EPS susceptibility in patients with comparable cumulative antipsychotic lifetime exposure.

After quality control, genotypes for 492,000 SNPs from 738 individuals remained for statistical analysis (10). In order to correct for population stratification we used the multidimensional scaling (MDS) approach implemented in PLINK (11). The first five dimensions captured the majority of the genetic substructure in CATIE. The GWAS was conducted using PLINK while incorporating the proxies for antipsychotic exposure and the MDS dimensions as covariates. We used a false discovery rate (FDR) to declare significance, where a $q$-value $<0.1$ declared genome-wide significance (12) and a $q$-value $<0.5$ to identify "potentially interesting" results. That is, the expected proportion of false discoveries among SNPs declared significant was 10\%/50\%. We also estimated the local FDR (13) to assess the (posterior) probability that our top findings were false discoveries.

\section{Results}

The proportion of individuals that at any time during the CATIE trial indicated some EPS symptoms (scale score $>0$ ) ranged from $72-82 \%$ for the three scales (Table 1). The mean number of assessments per genotyped subject was 7.5. Average sums for the most clinically relevant items, for each scale, are given in Table 1.

All GWAS p-values can be downloaded from www.pharmacy.vcu.edu/biomarker. Quantilequantile plots showing the distribution of the $p$-values are shown in Figure S1. These plots indicate that in general the $p$-values follow the expected null distribution indicated by the strait diagonal line. A few markers in the upper right corner deviate from this line suggesting a possible association. Three signals reached genome-wide significance according to our predefined threshold of $q$-value $<0.1$. Fourteen signals were potentially interesting using $q$ - 
values $<0.5$ as a threshold (Table 2). For SAS we detected genome-wide significance with rs17022444 $\left(p=1.2 \times 10^{-10}, q=4.8 \times 10^{-5}\right)$ located in an intergenic region on chromosome 2 p12 as well as with rs2126709 $\left(p=3.8 \times 10^{-7}, q=0.06\right)$ located in the 3 ' untranslated region (UTR) of zinc finger protein 202 (ZNF202) on chromosome 11q24. The third genome-wide significant signal involved the AIMS and rs7669317 $\left(p=7.7 \times 10^{-8}, q=0.05\right)$. Rs7669317 is located $167 \mathrm{~kb}$ from pyrophosphatase (inorganic) 2 (PPA2) and $16 \mathrm{~kb}$ from the locus encoding hypothetical protein FLJ20184 on chromosome 4q24. Regional plots for the significant results and Manhattan plots of the complete GWAS results can be found in Figures S2 and S3. No genome-wide significant signals were detected for the BARS and probable TD.

Secondary analyses of the genome-wide significant signals showed that the proportion of the variance explained by each SNP was similar in European and African Americans, that multimarker (haplotype) tests did not improve $p$-values, and that the detected signals were unlikely the result of genotyping errors.

\section{Discussion}

We performed GWAS to search for genetic susceptibility to EPS. Three signals reached genome-wide significance according to our predefined threshold. For each of these markers the posterior probability indicated a reasonable chance of a true finding. One of the SNPs, rs2126709, was located in the 3'UTR of ZNF202. This geneis a transcriptional repressor, controlling promoter elements found in for example apolipoprotein $\mathrm{E}$ ( $a p o E)$, lipoprotein lipase $(L P L)$, ATP binding cassette $(A B C)$ transporters $\mathrm{A} 1$ and $\mathrm{G} 1$, proteolipid protein $(P L P)$ and early growth response 3 (EGR3) (14). ZNF202 has been linked to dyslipidemia, high-density lipoprotein control, and many genes under its control are involved in reverse cholesterol transport (15). Considering that, next to adiposity tissue, the brain has the highest lipid content and that the myelin sheets surrounding and protecting neurons consists of approximately $80 \%$ lipids, a well functioning regulation of the lipid metabolism is of particular importance for brain function. Furthermore, demyelination can lead to diseases with ataxia and other movement problems, such as multiple sclerosis. PLP1, which is also under the transcriptional control of $Z N F 202$, is the predominant myelin protein present in the central nervous system. This gene has been associated with Pelizaeus-Merzbacher disease (16) in which involuntary movements, including parkinsonism, are among the major occurring symptoms. In addition, decreased mRNA expression of PLP1 has been observed in patients diagnosed with schizophrenia (17). Finally, EGR3 is expressed in a variety of cell types and plays a key role in nervous system development and plasticity. Mice lacking this gene have shown sensory ataxia and muscle spindle agenesis (18).

Markers rs17022444 and rs7669317 that were associated with SAS and AIMS, respectively, were not located in any known or predicted genes. Although this makes a false positive finding more likely, it does not preclude a true finding. Examples exist of intergenic associations replicating in independent samples (19) and there is considerable experimental evidence for long range regulatory effects mediated by these genomic regions (20).

Our findings require replication and validation. However, the present study demonstrates the potential of GWAS in order to discover genes and pathways that potentially mediate the adverse effects of antipsychotic medication. A better understanding of these mechanisms and the role of specific polymorphisms could eventually help to tailor individualized antipsychotic medication with minimal toxicity for patients with schizophrenia. 


\section{Supplementary Material}

Refer to Web version on PubMed Central for supplementary material.

\section{Acknowledgments}

The CATIE project was supported by NIMH contract N01 MH90001. Dr Sullivan was supported by R01s MH074027 and MH077139 and Dr van den Oord was supported by R01s MH078069 and HG004240.

\section{References}

1. Correll CU, Schenk EM. Tardive dyskinesia and new antipsychotics. Curr Opin Psychiatry. 2008; 21:151-156. [PubMed: 18332662]

2. Muller DJ, Schulze TG, Knapp M, Held T, Krauss H, Weber T, et al. Familial occurrence of tardive dyskinesia. Acta Psychiatr Scand. 2001; 104:375-379. [PubMed: 11722319]

3. Arranz MJ, de Leon J. Pharmacogenetics and pharmacogenomics of schizophrenia: a review of last decade of research. Mol Psychiatry. 2007; 12:707-747. [PubMed: 17549063]

4. Bakker PR, van Harten PN, van Os J. Antipsychotic-induced tardive dyskinesia and polymorphic variations in COMT, DRD2, CYP1A2 and MnSOD genes: a meta-analysis of pharmacogenetic interactions. Mol Psychiatry. 2008; 13:544-556. [PubMed: 18180754]

5. Zai CC, De Luca V, Hwang RW, Voineskos A, Muller DJ, Remington G, et al. Meta-analysis of two dopamine D2 receptor gene polymorphisms with tardive dyskinesia in schizophrenia patients. Mol Psychiatry. 2007; 12:794-795. [PubMed: 17767146]

6. Lieberman JA, Stroup TS, McEvoy JP, Swartz MS, Rosenheck RA, Perkins DO, et al. Effectiveness of antipsychotic drugs in patients with chronic schizophrenia. N Engl J Med. 2005; 353:1209-1223. [PubMed: 16172203]

7. Simpson GM, Angus JW. A rating scale for extrapyramidal side effects. Acta Psychiatr Scand Suppl. 1970; 212:11-19. [PubMed: 4917967]

8. Barnes TR. A rating scale for drug-induced akathisia. Br J Psychiatry. 1989; 154:672-676. [PubMed: 2574607]

9. Guy W. Abnormal invoulantary movement scale (AIMS). Alcohol, drug abuse and mental health administration. National Institute of Mental Health. 1976

10. Sullivan PF, Lin D, Tzeng JY, van den Oord E, Perkins D, Stroup TS, et al. Genomewide association for schizophrenia in the CATIE study: results of stage 1. Mol Psychiatry. 2008; 13:570-584. [PubMed: 18347602]

11. Purcell S, Neale B, Todd-Brown K, Thomas L, Ferreira MA, Bender D, et al. PLINK: a tool set for whole-genome association and population-based linkage analyses. Am J Hum Genet. 2007; 81:559-575. [PubMed: 17701901]

12. Storey JD. The positive false discovery rate: A Bayesian interpretation and the q-value. Annals of Statistics. 2003; 31:2013-2035.

13. Bukszar J, McClay J, van den Oord E. Estimating the posterior probability that genomewide association findings are true or false. Bioinformatics. $2009 \mathrm{Jul} 14$.

14. Schmitz G, Heimerl S, Langmann T. Zinc finger protein ZNF202 structure and function in transcriptional control of HDL metabolism. Curr Opin Lipidol. 2004; 15:199-208. [PubMed: 15017363]

15. Wagner S, Hess MA, Ormonde-Hanson P, Malandro J, Hu H, Chen M, et al. A broad role for the zinc finger protein ZNF202 in human lipid metabolism. J Biol Chem. 2000; 275:15685-15690. [PubMed: 10748193]

16. Willard HF, Riordan JR. Assignment of the gene for myelin proteolipid protein to the $\mathrm{X}$ chromosome: implications for X-linked myelin disorders. Science. 1985; 230:940-942. [PubMed: 3840606]

17. Aberg K, Saetre P, Jareborg N, Jazin E. Human QKI, a potential regulator of mRNA expression of human oligodendrocyte-related genes involved in schizophrenia. Proc Natl Acad Sci U S A. 2006; 103:7482-7487. [PubMed: 16641098] 
18. Tourtellotte WG, Milbrandt J. Sensory ataxia and muscle spindle agenesis in mice lacking the transcription factor Egr3. Nat Genet. 1998; 20:87-91. [PubMed: 9731539]

19. Zeggini E, Weedon MN, Lindgren CM, Frayling TM, Elliott KS, Lango H, et al. Replication of genome-wide association signals in UK samples reveals risk loci for type 2 diabetes. Science. 2007; 316:1336-1341. [PubMed: 17463249]

20. Pennacchio LA, Ahituv N, Moses AM, Prabhakar S, Nobrega MA, Shoukry M, et al. In vivo enhancer analysis of human conserved non-coding sequences. Nature. 2006; 444:499-502. [PubMed: 17086198] 


\section{Table 1}

Descriptive data for 738 genotyped CATIE subjects

\begin{tabular}{lc}
\hline Description & Proportion of ind. \\
\hline Males & $74 \%$ \\
Probable TD & $29 \%$ \\
AIMS score > & $72 \%$ \\
BARS score > & $75 \%$ \\
SAS score > & $82 \%$ \\
& Mean (s.d) \\
\cline { 2 - 2 } Age (in years) & $40.9(11.0)$ \\
Years treated & $16.7(11.2)$ \\
Years medicated & $14.3(10.8)$ \\
AIMS (item 1-7) & $1.48(2.33)$ \\
BARS (item 1-3) & $0.89(1.07)$ \\
SAS (item 1-6) & $0.21(0.25)$ \\
\hline
\end{tabular}

Means for AIMS, BARS, SAS are the summed item scores, for the clinically most relevant items (see Supplementary Materials for details), averaged across the multiple observations during the trials. 
